\title{
Understanding and countering the spread of conspiracy theories in social networks: Evidence from epidemiological models of Twitter data
}

\author{
Julian Kauk ${ }^{1 *}$, Helene Kreysa ${ }^{1}$, Stefan R. Schweinberger ${ }^{1,2}$ \\ 1 Department of General Psychology and Cognitive Neuroscience, Friedrich Schiller \\ University Jena, Am Steiger 3/1, 07743 Jena, Germany \\ 2 DFG Research Unit Person Perception, Friedrich Schiller University Jena, Jena, \\ Germany \\ * julian.kauk@uni-jena.de
}

\begin{abstract}
Conspiracy theories in social networks are considered to have adverse effects on individuals' compliance with public health measures in the context of a pandemic situation. A deeper understanding of how conspiracy theories propagate through social networks is critical for the development of countermeasures. The present work focuses on a novel approach to characterize the propagation of conspiracy theories through social networks by applying epidemiological models to Twitter data. A Twitter dataset was searched for tweets containing hashtags indicating belief in the "5GCoronavirus" conspiracy theory, which states that the COVID-19 pandemic is a result of, or enhanced by, the enrollment of the $5 \mathrm{G}$ mobile network. Despite the absence of any scientific evidence, the "5GCoronavirus" conspiracy theory propagated rapidly through Twitter, beginning at the end of January, followed by a peak at the beginning of April, and ceasing/disappearing approximately at the end of June 2020. An epidemic SIR (Susceptible-Infected-Removed) model was fitted to this time series with acceptable model fit, indicating parallels between the propagation of conspiracy theories in social networks and infectious diseases. Extended SIR models were used to simulate the effects that two specific countermeasures, fact-checking and tweet-deletion, could have had on the propagation of the conspiracy theory. Our simulations indicate that fact-checking is an effective mechanism in an early stage of conspiracy theory diffusion, while tweet-deletion shows only moderate efficacy but is less time-sensitive. More generally, an early response is critical to gain control over the spread of conspiracy theories through social networks. We conclude that an early response combined with strong fact-checking and a moderate level of deletion of problematic posts is a promising strategy to fight conspiracy theories in social networks. Results are discussed with respect to their theoretical validity and generalizability.
\end{abstract}

\section{Introduction}

The COVID-19 pandemic has been accompanied by an emerging stream of misinformation in social networks 11. The World Health Organization (WHO) explicitly noted the need to manage the "infodemic", i.e. to avoid a state of overabundance of information [2]. While (true) news play an important role in informing the public, misinformation can undermine the public health responses and can therefore 
significantly affect adherence to hygiene recommendations and efficacy of countermeasures $[3$. The effects of misinformation on pandemic-related outcome measures like incidence or mortality remain to be estimated, but it is reasonable to assume an adverse impact on both the spread of severe acute respiratory syndrome coronavirus 2 (SARS-CoV-2) [4] and efficient public health countermeasures [5]. When targeting misinformation, it is essential to consider social networks, as they have been shown to be important amplifiers [6]. There is an emerging body of research about the diffusion and prevalence of misinformation within social networks 7 . With respect to Twitter, Bovet and Maske 8 demonstrated for data from the 2016 US presidential election that the percentage of tweets containing misinformation can be up to $25 \%$. Similar fake news on this occasion were also spread via other social media such as Facebook [9]. Shin et al. [10] investigated the temporal dynamics of rumors on Twitter, revealing that false political rumors seem to reappear, whereas true rumors disappear after a short time period. More precisely, false political rumors had an average of 3.31 peaks whereas true rumors seem to appear only once. Consequently, true rumors showed significant "burstiness", meaning that nearly half of the total tweet volume ( $49.58 \%$ on average) was observed on a single day. There is also evidence that "echo chambers", i.e. the formation of groups where a shared belief is framed and reinforced 11], play a significant role in the amplification of misinformation 12. Even though social media platforms have put effort into updating their algorithms in order to limit the spread of misinformation, misinformation remains a constant source of problems: negative effects on adherence, democracy and diversification can be expected 13], which are potentially pervasive and long-lasting.

A better understanding of how misinformation propagates through social networks is a critical ingredient, since improper countermeasures may fail to work effectively. The idea that the spread of rumors can be modeled within an epidemiological framework emerged for the first time in a comment in 1964 [14], pointing out that infectiological states within an epidemiological SIR model (S: Susceptible, I: Infected and R: Removed) can be reinterpreted to fit for rumors. More recent works, for instance from Jin et al. [15, showed that more complex, adopted models may be more precise in describing rumor diffusion in social networks. Jin et al. used a SEIZ model (S: Susceptible, E: Exposed, I: Infected and Z: Skeptic; see also [16]) to characterize the spread of both (true) news and rumors on Twitter. They found that (i) their SEIZ model fitted better to the data than a simple SI model (S: Susceptible, I: Infected) and (ii) (true) news and rumors could be distinguished on the basis of the estimated model parameters. In general, previous research (see e.g. [17, 20]) has focused on model identification, aiming to identify epidemiological models which can characterize the diffusion of misinformation in social networks accurately.

The focus of the present study, however, is to simulate how countermeasures could affect the diffusion of misinformation in social networks. Therefore, we studied the "5GCoronavirus" conspiracy theory that emerged in January 2020 and stated that the spread of SARS-CoV-2 is caused/enhanced by the enrollment of the 5G mobile network 21, for a detailed description]. Tragically, this conspiracy theory was not restricted to social networks but apparently caused substantial physical damage, as more than 70 mobile phone masts were attacked by supporters in the United Kingdom alone 22,23 for two newspaper reports about this issue]. This damage to critical telecommunication infrastructure was preceded by an escalating situation on social networks, where users were called on to "break down" the $5 \mathrm{G}$ masts 24. It is reasonable to assume that countermeasures targeting this conspiracy theory on social networks could have reduced or even prevented the vandalism. Accordingly, we here studied how post deletion and fact-checking could have modulated the diffusion of the conspiracy theory in social networks. 
There is ample evidence that social media platforms rely on fact-checking: Facebook, for instance, explicitly describes its fact-checking strategy [25], and studies indicate that fact-checking on Facebook can be moderately beneficial 26 28]. Twitter uses labels and contextual cues to address the problem of misinformation [29] and announced in January 2021 that a "community-based approach to misinformation", namely "birdwatch", will be tested from now 30]. However, whether or not fact-checking is a sufficient tool to contain the spread of misinformation in social networks remains an open question [31]. We here understand fact-checking in a broadsense, meaning that the facts disproving a conspiracy theory are presented to the users in a preventive fashion, thus resembling more general public health communication.

In addition, the deletion of posts may be a promising tool as well [32], although there is evidently an indistinct line between responsible post deletion and censorship. If post deletion is applied circumspectly and in accordance with appropriate use policies, there might be acceptance for this countermeasure within the social media communities. Recent advances in the detection of misinformation on social networks 32 , for instance] may constitute a crucial ingredient to address the problem and can be considered as a necessary condition to perform both fact-checking and post deletion effectively.

We used advanced epidemiological models on a Twitter dataset in order to identify conditions under which countermeasures could have attenuated the spread of the conspiracy theory effectively. More precisely, we formulated a basic epidemiological model in order to characterize the diffusion of the "5GCoronavirus" conspiracy theory through Twitter. Subsequently, we incorporated both fact-checking and tweet deletion as well as a response lag into the basic epidemiological model, aiming to build up evidence about whether or not these countermeasures would have been capable of stopping the spread of the "5GCoronavirus" conspiracy theory through Twitter.

\section{Methods}

\section{Basic epidemiological model}

We used a simple SIR compartment model (without vital dynamics) 36 to characterize the propagation of the "5GCoronavirus" conspiracy theory. Although other authors have suggested different models to characterize the dynamics of misinformation diffusion through social networks [15,37, for instance], we decided to use the SIR model because (i) it is less complex and deals with only five parameters, (ii) it may constitute a good starting point to incorporate countermeasures without creating complex interactions, and (iii) other researchers have used the SIR model for the same purpose as well [18,20]. The SIR model flow can be described by

$$
S \stackrel{\frac{\beta S I}{N}}{\longrightarrow} I \stackrel{\alpha I}{\longrightarrow} R
$$

where compartments $S(t), I(t)$, and $R(t)$ sum up to the total population $N, \beta$ is the infection rate, and $\alpha$ is the recovery rate. The greater $\beta$, the more likely an infected individual infects at least one susceptible individual. The smaller $\alpha$, the longer an individual remains infectious and not recovered (infection period is given by $\frac{1}{\alpha}$ ). The dynamics of the model can be characterized by a set of ordinary differential equations (ODEs; 38). The set of ODEs is given by 


$$
\begin{aligned}
\frac{d S}{d t} & =-\frac{\beta S I}{N} \\
\frac{d I}{d t} & =\frac{\beta S I}{N}-\alpha I \\
\frac{d R}{d t} & =\alpha I .
\end{aligned}
$$

As shown in Table1 (top and middle row), the SIR model can be redefined fairly easily to apply to conspiracy theories. However, the strict redefinition of the R compartment is problematic, as there is no immune system "curing" false beliefs. In consequence, the $\mathrm{R}$ compartment needs further elaboration. It is reasonable to assume that the cognitive and communicative efforts an individual puts into a conspiracy theory both decline after a given period of time due to forgetting, habituation or decreasing interest 39 41. We therefore assume that the conspiracy theory is out of mind after a given time period, and consequently the individual progresses to the $\mathrm{R}$ compartment. With respect to the propagation of conspiracy theories specifically on Twitter, a few more aspects had to be considered in the definition of the model. Susceptible individuals are considered to be susceptible to a conspiracy theory, but have not posted a corresponding tweet yet. Infected individuals are considered to believe a conspiracy theory, indicated by having posted a corresponding tweet. Table 1 (bottom row)

\begin{tabular}{|c|c|c|c|c|}
\hline \multirow[b]{2}{*}{ Scope } & \multirow[b]{2}{*}{$\mathrm{N}$} & \multicolumn{3}{|c|}{ Compartments } \\
\hline & & $\mathrm{S}$ & I & $\mathrm{R}$ \\
\hline Epidemiology & $\begin{array}{l}\text { total popula- } \\
\text { tion }\end{array}$ & $\begin{array}{l}\text { susceptible to } \\
\text { an infectious } \\
\text { disease }\end{array}$ & being infected & $\begin{array}{l}\text { removed due } \\
\text { to immunity } \\
\text { or death }\end{array}$ \\
\hline $\begin{array}{l}\text { Conspiracy } \\
\text { Theory (in } \\
\text { general) }\end{array}$ & $\begin{array}{l}\text { total popula- } \\
\text { tion }\end{array}$ & $\begin{array}{l}\text { susceptible to } \\
\text { a conspiracy } \\
\text { theory }\end{array}$ & $\begin{array}{l}\text { believing con- } \\
\text { spiracy theory }\end{array}$ & $\begin{array}{l}\text { not believing } \\
\text { conspiracy } \\
\text { theory any- } \\
\text { more }\end{array}$ \\
\hline $\begin{array}{l}\text { Conspiracy } \\
\text { Theory } \\
\text { (Twitter) }\end{array}$ & $\begin{array}{l}\text { Theoretically: } \\
\text { All active } \\
\text { twitter users; } \\
\text { De facto: Un- } \\
\text { clear (e.g. } \\
\text { 15]) }\end{array}$ & $\begin{array}{l}\text { have not } \\
\text { posted a sup- } \\
\text { porting tweet }\end{array}$ & $\begin{array}{l}\text { have posted } \\
\text { a supporting } \\
\text { tweet }\end{array}$ & $\begin{array}{l}\text { conspiracy } \\
\text { the- } \\
\text { ory is out of } \\
\text { mind/forgotten } \\
3941\end{array}$ \\
\hline
\end{tabular}
summarizes the SIR model adapted for Twitter.

Table 1. Redefining the SIR model for conspiracy theories.

\section{Extended epidemiological models}

In order to incorporate potential countermeasures into the basic SIR model, we identified three extended SIR models which include deletion of tweets, fact-checking and both countermeasures combined. Additionally, in order to account for delays in the beginning of countermeasures, we introduced the parameter $\delta$, which reflects the delay until countermeasures are implemented. The following sections provide a detailed formulation and description of these extended SIR models. Note that, if not indicated otherwise, the parameters (and their definitions) of the basic SIR model (described above) apply to all extended SIR models. 


\section{SIR model with fact-checking}

The SIR model with fact-checking $\left(S I R_{\text {fact-checking }}\right)$ extends the basic SIR model by the parameter $\gamma$, representing a constant ratio of individuals progressing directly from compartment $S$ to $R$ per time unit. The assumption of a constant ratio is simplistic, but may hold for relatively small values of $\gamma$, as it is reasonable to assume that there is a significant proportion of susceptible individuals who are responsive to fact-checking. In order to consider the delay parameter $\delta$ in the model, we introduce the variable $\gamma_{\delta}$, which is a function of $t$ and $\delta$ and is "activated" only if the delay $\delta$ is exceeded. This variable is given by

$$
\gamma_{\delta}= \begin{cases}\gamma & \text { if } t>\delta \\ 0 & \text { otherwise }\end{cases}
$$

The flow of the model therefore can be written as

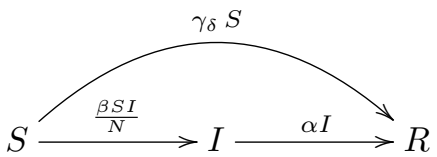

and the set of ODEs is given by

$$
\begin{aligned}
\frac{d S}{d t} & =-\frac{\beta S I}{N}-\gamma_{\delta} S \\
\frac{d I}{d t} & =\frac{\beta S I}{N}-\alpha I \\
\frac{d R}{d t} & =\alpha I+\gamma_{\delta} S .
\end{aligned}
$$

Accordingly, $\gamma$ controls how effectively susceptible individuals can be prevented from becoming convinced by a conspiracy theory.

\section{SIR model with tweet deletion}

The SIR model with tweet deletion $\left(S I R_{\text {deletion }}\right)$ is similar to the basic SIR model except for an additional parameter $\zeta$, representing a constant ratio of individuals being moved from compartment $I$ to compartment $R$ per time unit. The assumption of a constant ratio is simplistic as well, but may resemble the limited capabilities of a social media platform to detect and delete suspect tweets. The definition of the variable $\zeta_{\delta}$ is equivalent to $\gamma_{\delta}$ except that $\gamma$ is replaced by $\zeta$. The variable is therefore given by

$$
\zeta_{\delta}= \begin{cases}\zeta & \text { if } t>\delta \\ 0 & \text { otherwise }\end{cases}
$$

The flow of the model is given by

$$
S \stackrel{\frac{\beta S I}{N}}{\longrightarrow} I \stackrel{\left(\alpha+\zeta_{\delta}\right) I}{\longrightarrow} R
$$

and the set of ODEs can be written as 


$$
\begin{aligned}
\frac{d S}{d t} & =-\frac{\beta S I}{N} \\
\frac{d I}{d t} & =\frac{\beta S I}{N}-\left(\alpha+\zeta_{\delta}\right) I \\
\frac{d R}{d t} & =\left(\alpha+\zeta_{\delta}\right) I .
\end{aligned}
$$

Parameter $\zeta$ therefore controls how many individuals who are convinced by a conspiracy theory move to the $R$ compartment by deletion of their tweets.

\section{SIR model with mixed countermeasures}

SIR model with mixed countermeasures $\left(S I R_{\text {mixed }}\right)$ combines $S I R_{\text {fact-checking }}$ and $S I R_{\text {deletion }}$ by introducing both $\gamma$ and $\zeta$ to the model. Note that the definitions of both parameters (and their corresponding variables $\gamma_{\delta}$ and $\zeta_{\delta}$ ) also apply to the $S I R_{\text {mixed }}$ model. The flow can be described by

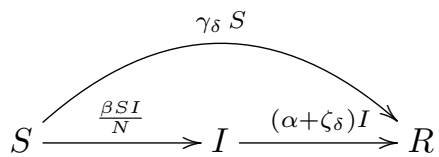

and the set of ODEs is given by

$$
\begin{aligned}
\frac{d S}{d t} & =-\frac{\beta S I}{N}-\gamma_{\delta} S \\
\frac{d I}{d t} & =\frac{\beta S I}{N}-\left(\alpha+\zeta_{\delta}\right) I \\
\frac{d R}{d t} & =\left(\alpha+\zeta_{\delta}\right) I+\gamma_{\delta} S .
\end{aligned}
$$

\section{Parameter Identification}

The following sections provide detailed information about the procedure of parameter identification for both the basic SIR model and the extended SIR models.

\section{Basic SIR model}

As both $\beta$ and $\alpha$ cannot be deduced properly from previous research, these parameters were treated as unknowns and had to be estimated empirically. These estimated parameters of the basic SIR model were also used subsequently to parametrize the extended SIR models. The total population size $N$ was also not specified in advance, as $N$ is theoretically given by any Twitter user who could have been exposed to the "5GCoronavirus" conspiracy theory, but de facto $N$ is not easy to estimate 15, 16, for a detailed reasoning]. Accordingly, the initial numbers of individuals in the compartments, $S\left(t_{0}\right), I\left(t_{0}\right), R\left(t_{0}\right)$, were treated as unknowns too.

Parameter Identification was done in $R$ [42, version 4.0.1 (2020-06-06)] using the EpiModel package 43 for model specification combined with the $R$ stats function optim for identification of optimal parameter values. A least squared criterion was applied as given by

$$
\hat{\theta}=\underset{\Theta}{\arg \min }\left(\sum_{i=0}^{t_{\max }}\left(I_{\text {cum }}\left(t_{i}\right)-\hat{I}_{\text {cum }}\left(t_{i}\right)\right)^{2}\right),
$$


where $\hat{\theta}$ refers to the vector of estimated parameter values, $\Theta$ is the parameter space, $\quad{ }_{170}$ $t_{\max }$ refers to the most recent date included in the analysis, $t_{i}$ is the $i$-th date, $I_{\text {cum }}\left(t_{i}\right){ }_{171}$ is the cumulated incidence up to $t_{i}$, and $\hat{I}_{c u m}\left(t_{i}\right)$ represents the cumulated incidence according to the model up to $t_{i}$.

In order to evaluate the basic SIR model, we report relative error in 2-norm as given by

$$
\epsilon_{\text {rel }}=\frac{\left\|I_{\text {cum }}(t)-\hat{I}_{\text {cum }}(t)\right\|_{2}}{\left\|I_{\text {cum }}(t)\right\|_{2}},
$$

as well as the mean absolute error $(M A E)$ described by

$$
M A E=\frac{\left\|I_{\text {cum }}(t)-\hat{I}_{\text {cum }}(t)\right\|_{1}}{n},
$$

where $n$ stands for the number of data points.

\section{Extended SIR models}

The identified parameter values of the basic SIR model were used to parametrize the extended SIR models. Consequently, the parametrized extended SIR models are similar to the parametrized basic SIR model, except for their additional parameters $\gamma, \delta$ and $\zeta$, where the respective parameter values were defined a priori. In order to identify realistic parameter values for $\gamma, \delta$ and $\zeta$, we had to take into account the time scale of the "5GCoronavirus" conspiracy theory, which is characterized by the onset $t_{0}$ at the end of January 2020 and an incidence peak $t_{I M a x}$ at the beginning of April 2020 24. In the interest of simplicity, we here elaborated three explicit levels for each parameter, even though the parameters are, in principle, considered to be continuous.

Countermeasures' delay $\delta$. It is reasonable to assume different tempi when considering the response of social networks to conspiracy theories. A response may be considered as Early, Delayed or Late. We here define an early response as two weeks from $t_{0}$, while a delayed response is defined as six weeks from $t_{0}$. A late response is peak-adjusted and defined as two weeks before $t_{I M a x}$. We therefore here studied three levels of $\delta$ as given by $\delta_{1}=14, \delta_{2}=42$ and $\delta_{3}=t_{I M a x}-14$.

Fact-checking parameter $\gamma$. The parameter $\gamma$ reflects how effectively individuals can be prevented from becoming convinced by the conspiracy theory by the implementation of fact-checking. We here assumed that only a small percentage of individuals in the $S$ compartment can be convinced per week to reject the conspiracy theory. There are reasonable arguments for this assumption: (i) Not every Twitter user uses Twitter everyday, (ii) fact-checking countermeasures may take some time to have an effect on Twitter users' minds, and (iii) Twitter users may show resistance to fact-checking 44, 45. Taking these considerations into account, we here tested three levels of $\gamma$ as given by $\gamma_{1}=\frac{0.01}{7}, \gamma_{2}=\frac{0.03}{7}$ and $\gamma_{3}=\frac{0.05}{7}$. Accordingly, if considering $\gamma_{1}=\frac{0.01}{7}$, approximately one percent of the $S$ compartment would progress to the $R$ compartment per week.

Tweet deletion parameter $\zeta$. The parameter $\zeta$ indicates how many individuals can be removed from the $I$ compartment by deleting their tweets. There is evidence that social networks differ in the extent to which they use fact-checking and delete posts 46. However, it is reasonable to assume that social networks have a fair chance of using (semi-)automatic software to detect suspect tweets. Large-scale deletion of posts is nonetheless difficult, as (i) debate and discussions are essential to social networks, (ii) suspect tweets can be false-positive, and (iii) users may undermine these countermeasures by using different accounts, phrases, hashtags et cetera. We therefore assume that social networks are able to perform tweet deletion at low to medium rates. 
Accordingly, we defined that $\zeta$ should have three levels with $\zeta_{1}=\frac{0.06}{7}, \zeta_{2}=\frac{0.12}{7}$ and $\zeta_{3}=\frac{0.25}{7}$.

214

\section{Data}

We used data collected by Banda et al. 47, who provide aggregated Twitter data beginning from the 1st of January 2020 ongoing until the time of writing (March 2021). They used the Twitter Stream API to search for specific pandemic-related keywords, e.g. "coronavirus", "covid19", "CoronavirusPandemic" et cetera; see [47, for the full list]. Due to Twitter terms of services they provide tweet ids only, meaning that the content of the tweets has to be downloaded separately.

On this basis, we conducted a large-scale download of tweets, requesting tweet content using the Twitter API from more than 140 million tweet ids. Tweets were downloaded and analyzed from January 1, 2020, until August 15, $2020\left(t_{\max }\right)$. We searched the downloaded tweets for the keyword " $5 \mathrm{G}$ " (case insensitive) and stored matching tweets in a dataset. However, an interim analysis to check whether the data was complete revealed that the downloaded data was substantially affected by data dropouts, as only approximately $40 \%$ of the requested tweets had been successfully downloaded. We do not know precisely the reason for $60 \%$ missing data, but it seems reasonable to assume that account and tweet deletions play an important role.

In contrast, the datasets provided by Banda et al. [47] should be far less affected by data dropouts, as they report that they update their dataset every two days.

Consequently, we decided to use their "hashtags" dataset, which stores the hashtags and their frequencies per day for all clean tweets. Clean tweets mean that retweets are not included. Even though the exclusion of retweets was not optimal for our research purpose, the "hashtags" dataset should nevertheless be a fair proxy for the true dynamics of the "5GCoronavirus" conspiracy theory on Twitter. Please note that the tweet recording period remains the same, i.e. from January 1, 2020, until August 15, 2020 .

In order to map these true dynamics, we identified ten hashtags which (i) clearly indicate that $5 \mathrm{G}$ is harmful or connected to SARS-CoV-2, and (ii) were most frequently used. Table 2 shows the selected hashtags and their frequencies.

Table 2. Frequency table of the ten most used hashtags indicating that 5G is harmful or connected to SARS-CoV-2.

\begin{tabular}{l|r}
\hline Hashtag & Frequency \\
\hline \#5gcoronavirus & 2171 \\
\#5gkills & 1311 \\
\#stop5g & 842 \\
\#5gcorona & 466 \\
\#wuhan5g & 228 \\
\#5gvirus & 213 \\
\#5gdeadlyweapon & 207 \\
\#no5g & 85 \\
\#saynoto5g & 43 \\
\#5gcononavirus & 45 \\
\hline \#(Sum) & 5611 \\
\hline
\end{tabular}




\section{Results}

\section{Descriptive Analysis}

In total, 5611 hashtags were recorded from $t_{0}$ to $t_{\max }$. The first hashtag was observed on January 27, $2020\left(t_{0}\right)$, which is in line with findings from Bruns et al. [24. Table 2 provides the frequency of each hashtag. The hashtag incidence time series is depicted in Fig 1 A, showing that the "5GCoronavirus" conspiracy theory began at the end of January, followed by an exponential increase with a peak at the beginning of April 2020 and ending approximately at the end of June 2020. The maximal hashtag incidence was observed on April 8, $2020\left(t_{\text {IMax }}\right)$. Fig 1 A also shows an interesting pattern of reappearing "incidence bursts", meaning that the "5GCoronavirus" conspiracy theory reappeared occasionally after $t_{I M a x}$. This observation is in line with the findings from Shin et al. 10], who reported that misinformation tend to come back after initial publication.

Fig 1. Acceptable correspondence of observed and predicted hashtag incidence. (A) Hashtag incidence over time. Please note that "Rolling mean" refers to the simple moving average with the rolling window $k=7$. (B) Best basic SIR model fit. (C) Model predictions of the compartment sizes over time.

\section{Basic SIR model}

Table 3 shows the estimated parameter values yielded by the parameter identification procedure, as well as the error norms $\epsilon_{r e l}$ and $M A E$. Fig $1 \mathrm{~B}$ and $\epsilon_{r e l}$ both indicate that the model fit of the basic SIR model is acceptable. The $\epsilon_{r e l}$ reported here is in line with results provided by Jin et al. [15], who found similar values of $\epsilon_{r e l}$ for their models. We therefore conclude that our basic SIR model shows fair correspondence of observed and predicted incidence.

Table 3. Parameters and parameter estimations of the best fit basic SIR model.

\begin{tabular}{l|l|l}
\hline Parameter & Meaning & Estimation \\
\hline$\beta$ & infection rate & 0.3 \\
$\alpha$ & recovery rate & 0.11 \\
$\frac{1}{\alpha}$ & infection period & approx. 9 \\
$\mathcal{R}_{0}=\frac{\beta}{\alpha}$ & basic reproduction number & 2.7 \\
\hline$S(0)$ & initial susceptible & 7060.94 \\
$I(0)$ & initial infectious & 1.1 \\
$R(0)$ & initial removed & 2936.53 \\
$N=S(0)+I(0)+R(0)$ & population size & approx. 9999 \\
\hline$\epsilon_{r e l}$ & relative error in 2-norm & 0.0561 \\
$M A E$ & mean absolute error & 194.52 \\
\hline
\end{tabular}

Fig $1 \mathrm{C}$ shows the model predictions of the SIR compartment sizes over time. According to these predictions, prevalence is maximal on April 20, 2020 with approx. 971 active cases. This prediction coincides roughly with newspaper reports that the majority of attacks on mobile phone masts were performed in the beginning / middle of April 2020 22. The model also shows that there was already a significant proportion of individuals who were recovered/removed at $t_{0}$, accounting for the fact that not all individuals are necessarily receptive to conspiracy theories. In fact, however, considering the ratio of $S(0)$ and $R(0)$, the model makes the assumption that a majority of 
individuals was potentially susceptible to the "5GCoronavirus" conspiracy theory. This seems unrealistic with respect to prior findings, which indicate that only a minority of people is sensitive to conspiracy theories per se [48, for instance]. Finally, this model implies that there was a significant proportion of individuals (approx. 23\%) who were susceptible but did not progress to the $I$ compartment, arguably resembling the effects of herd immunity.

\section{Extended SIR models}

We compared the extended SIR models by plotting the incidence curves of all $\left(S I R_{\text {fact-checking }}\right.$ and $\left.S I R_{\text {deletion }}\right)$ parameter combinations, and of corresponding parameter combinations for the $S I R_{\text {mixed }}$ models. We also show the incidence curve of the basic SIR model as the baseline model. Fig 2 shows the resulting matrix. Please note that the basic SIR model is also referred to as "baseline SIR model" in the following sections.

Fig 2. Predicted incidence over time across different extended SIR models and parameters. Note that different levels of $\delta$ are depicted column-wise, while different extended SIR models are depicted row-wise. Different levels of $\gamma$ and $\zeta$ vary within each panel A-I. Please also note that, in the interest of simplicity, only corresponding parameter combinations, i.e. $\left(\gamma_{1}, \zeta_{1}\right),\left(\gamma_{2}, \zeta_{2}\right)$ and $\left(\gamma_{3}, \zeta_{3}\right)$, are depicted.

Furthermore, in order to evaluate the extended SIR models in a more quantitative fashion, we calculated the incidence proportion $\operatorname{IP}\left(t_{\max }\right)$, which reflects the probability that a susceptible individual will become infected up to $t_{\max }$. Accordingly, this measure characterizes the percentage of susceptible individuals who become infected over the whole time period. We here denote this measure as $I P_{f}$. Fig 3 shows heatmaps of $I P_{f}$ given different parameters on the axes. Please note that the $I P_{f}$ of the baseline SIR model is 0.77 , meaning that $77 \%$ of the susceptible individuals get infected over time in the baseline SIR model.

Fig 3. Heatmaps of $I P_{f}$ for different parameter combinations. Please note that A and B do not share the same metric, i.e. "blue" in A does not correspond to "blue" in B with respect to $I P_{f}$. Please also note that panel $\mathrm{C}$ shows the heatmaps of $\zeta \times \gamma$ given different levels of $\delta$.

\section{SIR model with fact-checking}

Fig 2A-C clearly indicates an effect of fact-checking on the incidence, which is more prominent for higher levels of $\gamma$. Accordingly, the more fact-checking is applied, the less individuals become convinced by the conspiracy theory. Importantly, the effect size is strongly $\delta$-dependent, meaning that fact-checking is most effective if applied in early stages (Fig 2A), while late fact-checking is nearly useless, irrespective of the $\gamma$ level (Fig $2 \mathrm{C}$ ). Considering the effects of an Early response combined with a high level of $\gamma$, the incidence curve shows a very moderate progression, indicating that fact-checking alone - if applied early - might have been able to contain the spread of the "5GCoronavirus" conspiracy theory. This observation is paralleled by the corresponding incidence proportion of $I P_{f}=0.16$ (see S1 Table), which provides further quantitative evidence for the superiority of this model over the baseline model. A Delayed response, meanwhile, shows significant losses in effectiveness of fact-checking compared to an Early response (see Fig $2 \mathrm{~B}$ ). 
These results are further supported by Fig $3 \mathrm{~A}$, which clearly indicates that fact-checking is effective only if applied early but becomes useless for higher values of $\delta$. Taking these findings together, we conclude that there is a narrow time window where fact-checking is extremely useful to flatten the incidence curve. Afterwards, the effect of fact-checking alone is substantially attenuated with nearly no effect.

\section{SIR model with tweet-deletion}

Fig 2D-F shows a substantial effect of tweet-deletion on the incidence, which seems to be essentially linearly related to $\zeta$. Interestingly, the effect size is not as $\delta$-dependent as was predicted for the SIR model with fact-checking. Another interesting feature shown in the incidence curves is that there is a slight positive time shift of the peaks for an Early response (Fig 2D), while an inverse pattern can be observed for a Late response (Fig 2F). This leads us to the conclusion that tweet-deletion has potentially a second positive effect in addition to incidence reduction, by prolonging the available response time to conspiracy theories in social networks, providing more time to introduce additional countermeasures. However, it seems that tweet-deletion alone would not have been a sufficient tool to control the spread of the "5GCoronavirus" conspiracy theory: Considering the case of an Early response with maximal tweet-deletion, the effect indicated by $I P_{f}=0.541$ is relatively weak, compared to the SIR model with fact-checking. The effects of tweet-deletion remain relatively stable when considering a Delayed response (Fig 2E), but the effect of the positive peak-shift vanishes almost completely.

These results are supported by Fig $3 \mathrm{~B}$, showing that the effect of tweet-deletion is relatively stable over time and declines significantly only in extremely late stages. Accordingly, there is a wider time window in which tweet-deletion can be applied profitably. However, this finding is weakened by the observation that tweet-deletion in general seems to have a limited potential for reducing the incidence, compared to fact-checking.

\section{SIR model with mixed countermeasures}

Finally, Fig 2G-I suggests that combining fact-checking and tweet deletion provides an effective tool to reduce the incidence substantially, even if the response is not Early. Considering Fig $2 \mathrm{H}$ (Delayed response), a strong response, as given by $\left(\gamma_{3}, \zeta_{3}\right)$, can flatten the curve to a moderate level. If both countermeasures are applied strongly in an Early stage (Fig $2 \mathrm{G}$ ), the incidence can be reduced to close to zero $\left(I P_{f}=0.029\right)$, presenting a powerful mechanism which would have been able to contain the spread of the "5GCoronavirus" conspiracy theory. Even an Early moderate response, as given by $\left(\gamma_{2}, \zeta_{2}\right)$, shows satisfying outcomes, as indicated by the incidence curve and the $I P_{f}$ of 0.204. Nonetheless, even the SIR model with mixed countermeasures fails, as did the previous extended SIR models, to provide evidence that the spread of the "5GCoronavirus" conspiracy theory could have been controlled in a Late stage. This is reflected in the incidence curves in Fig 2I. It seems, however, as there is at least some "damage control", as a strong response shows a $I P_{f}$ of 0.408 , which is a significant reduction compared to the baseline SIR model with $I P_{f}=0.77$.

Fig $3 \mathrm{C}$ further underpins that the effect of countermeasures is highly time-sensitive. While there is a relatively large space of parameter combinations with good outcomes ("blue" regions) in the left panel (Early response), there is only a small "blue" region left when considering a Delayed response (panel in the middle). Accordingly, stronger countermeasures are needed if the response is Delayed to achieve the same outcome. The right panel of Fig $3 \mathrm{C}$ shows that a Late response can only inadequately control the 
incidence. Fig $3 \mathrm{C}$ also shows the $\delta$-dependency of both parameters, $\gamma$ and $\zeta$, as $\gamma$ is effective only if applied early, while $\zeta$ is less time-sensitive.

\section{Discussion}

We here demonstrated that the spread of conspiracy theories can be characterized using epidemiological models. This finding is in line with previous research 15, 20, further supporting the potential of epidemiological models, even outside their original scope of infectious diseases (see [49,50, for instance] and [51, for an overview]). Furthermore, we evaluated the effects that countermeasures could have had on the propagation of the "5GCoronavirus" conspiracy theory on Twitter. Our simulations indicated that (i) fact-checking is an effective mechanism in an early stage of conspiracy theory diffusion, (ii) tweet-deletion shows moderate efficacy and is less time-sensitive than fact-checking, and (iii) combined countermeasures constitute a promising tool to effectively limit conspiracy theory diffusion. Our simulations also clearly show that response time is critical when dealing with conspiracy theories in social networks. Taking these findings together, we conclude that an early response combined with strong fact-checking and a moderate level of tweet deletion is necessary to control the diffusion of a conspiracy theory through a social network.

The data we have analyzed here potentially implies some limitations with respect to the validity of the underlying epidemiological models. As stated above, we had to use hashtag data instead of data on an individual level. That means that we - strictly speaking - modeled the diffusion of hashtags, but not the infection curve of individuals. However, it seems reasonable to assume that hashtag incidence is a fair proxy of the "true" incidence curve. Previous research suggests that hashtags can be modeled by epidemiological models too [20]. At the same time, it is also important to note that false positive tweets may play a significant role too: An individual who tweeted a suspect hashtag ("5GCoronvarirus", for instance) need not necessarily be convinced by the conspiracy theory, but could use the hashtag in a humoristic fashion, or could state that there is no evidence for the conspiracy theory. Our interim analysis of the downloaded tweets also supports the presence of false positive tweets, indicating that future studies should make efforts to "filter out" these tweets by applying text classification. Text classification of tweets has been intensively studied with respect to sentiment analysis 52, showing that sentiment analysis is very challenging due to the extreme heterogeneity of the tweets and limited length (max. 280 characters) of the tweets. There is also research about the detection of misinforming tweets [53 54], indicating the potential of machine learning to estimate tweet credibility. Associated with the problem of false positive tweets are social bots, which evidently facilitate the spread of misinformation through social networks 55 but do not constitute human entities. However, the application of machine learning and respective tools for both the detection of misinforming tweets 56, for instance] and bots [57] seems reasonable and may help to better understand the "true" diffusion of misinformation through social networks.

There is also a need for further theoretical and empirical work in order to produce more valid models of epidemiological conspiracy theory diffusion in social networks. Our simple SIR model showed acceptable model fit, but it failed to account for both the extreme peak at the beginning of April 2020 and the reappearing "incidence bursts". More complex models may provide solutions to the weaknesses of the simple SIR model. However, even this simple model was generally able to characterize the propagation of the "5GCoronavirus" conspiracy theory. Future studies should focus on (i) improving the underlying epidemiological model, (ii) advancing the parameter identification procedure [58, for an infomrative article about this issue], and (iii) increasing the number of studied conspiracy theories. Implementing these points may help to learn 
more about the underlying processes of when and how conspiracy theories propagate through social networks.

Taking into account recent advances in memory and human factors research $[59$, for instance] may also help to improve the underlying epidemiological model: Ognyanova et al. 60 found that fake news exposure was associated with a decline in mainstream media trust. Transferring this finding to the epidemiological model would imply that there exists a significant proportion of susceptible individuals who are unresponsive to fact-checking at all. There is also evidence that if an individual has once established a false belief, "curing" it can be extremely challenging [61, meaning that - within an epidemiological framework - infected individuals should not be seen as recovered after the infection period, but potentially sensitive to become "infectious" again. In this context, it is interesting to note that individuals who believe in one conspiracy theory are also more likely to believe other, unrelated rumors 62. Implementing such empirical findings into the model may prove challenging, but would increase their ability to adequately reflect real-world relationships.

More generally, the extended SIR models used in this study should be seen as starting points with the need of further theoretical elaboration and empirical work. For instance, the values of the parameter $\gamma$ were defined arbitrarily, as it is unclear how effective fact-checking actually is. Furthermore, it is unlikely that fact-checking affects susceptible individuals only: Conceivably, fact-checking could also convince an infected individual to reject the conspiracy theory in question or motivate removed individuals to convince susceptible and infected individuals that the conspiracy theory in question is wrong. One more important point is that the analyzed Twitter data potentially already includes the effects of fact-checking and tweet deletion: Twitter evidently deleted tweets which called for a "breakdown" of 5G towers 29. While our results suggest that implementing such measures may be effective in countering the spread of conspiracy theories, the loss of data through deletion poses a problem for gaining a clear picture of individuals' usage behaviour of the corresponding hashtags. Projects such as "FakeNewsNet" 63 or "FacebookHoax" 33, which provide large-scale datasets of fake news posts before the introduction of countermeasures, can potentially address this problem, especially as the recorded fake news posts are extremely heterogeneous with respect to the underlying fake news story.

The present findings, that fact-checking and tweet-deletion seem to differ in their efficiency as countermeasures against misinformation, should be interpreted against psychological models of belief formation and human memory - especially those that consider psychological mechanisms for the formation and persistence of false memories or false knowledge 64, 65. For instance, the relative inefficacy of fact-checking at later stages of spread might indicate that to be effective, fact-checking needs to take place before much memory consolidation of misinformation has occurred. Our findings are also potentially relevant for the increasingly urgent question of how to combat deliberate misinformation attacks [59] that are solely intended to support the goals of the attacker, and that are increasingly implemented via automated bots.

Arguably, misinformation attacks unfold their deceptive efficiency via basic psychological mechanisms. For instance, in a digital world with many competing pieces of information, more salient bits of information grab more attention, which could explain in part why false stories are much more likely to be retweeted on Twitter than true stories [66]. Early (mis)information also contributes to establishing an "anchor" - a mental model against which subsequent incoming information is interpreted; once a belief is established, a confirmation bias may influence the belief holder to actively seek information that confirms the belief, and to discount information that is inconsistent with the belief (for a model of cognitive mechanisms involved in processing misinformation attacks, see [59]). As misinformation attacks are becoming more 
sophisticated and dangerous, the design and scientific evaluation of countermeasures, though currently in its infancy, is becoming a high-priority challenge for society.

Immediate and repeated communication of true facts is probably more efficient than the repeated correction of wrong information, but far more research is needed to translate the emerging cognitive psychology of information attacks into an efficient and versatile set of defensive measures [59,67. Increasing evidence on the negative social consequences of conspiracy theories, e.g., for adherence to pandemic restrictions and vaccination recommendations, for the intention to engage in politics or for reducing one's carbon footprint 68, underlines that efforts towards science-based solutions for addressing misinformation attacks may not only be well-invested, but ultimately vital.

\section{Conclusion}

The present study has provided important evidence for the effects that countermeasures can have on the propagation of conspiracy theories through social networks. We found that fact-checking is an extremely powerful tool in the early stages of conspiracy theory diffusion, but fails in later stages. Tweet-deletion is less powerful, but also less time-dependent than fact-checking. We therefore conclude that fact-checking is the better choice if the conspiracy theory is not in an exponential increase yet. Tweet-deletion should be applied strongly if the conspiracy theory is already propagating rapidly through the social network, and moderately otherwise. In view of the difficulty of identifying novel conspiracy theories, a combined implementation of multiple counter-measures is most likely to prove successful.

\section{S1 Table. Outcomes of the tested extended SIR models.}

\section{Acknowledgments}

We thank Thoraf Kauk for providing additional computational resources. We also thank Christine Nussbaum and Frank Nussbaum for their comments on the methods section of the manuscript.

\section{References}

1. Apuke OD, Omar B. Fake news and COVID-19: modelling the predictors of fake news sharing among social media users. Telematics and Informatics. 2020; p. 101475 .

2. World Health Organization et al . Novel Coronavirus (2019-nCoV): situation report, $13 ; 2020$.

3. Simonov A, Sacher SK, Dubé JPH, Biswas S. The persuasive effect of fox news: non-compliance with social distancing during the covid-19 pandemic. National Bureau of Economic Research; 2020.

4. Tasnim S, Hossain MM, Mazumder H. Impact of rumors and misinformation on COVID-19 in social media. Journal of preventive medicine and public health. 2020;53(3):171-174.

5. Dickmann P, Biedenkopf N, Keeping S, Eickmann M, Becker S. Risk communication and crisis communication in infectious disease outbreaks in Germany: what is being done, and what needs to be done. Disaster medicine and public health preparedness. 2014;8(3):206-211. 
6. Cinelli M, Quattrociocchi W, Galeazzi A, Valensise CM, Brugnoli E, Schmidt AL, et al. The covid-19 social media infodemic. arXiv preprint arXiv:200305004. 2020;

7. Tandoc Jr EC. The facts of fake news: A research review. Sociology Compass. 2019;13(9):e12724.

8. Bovet A, Makse HA. Influence of fake news in Twitter during the 2016 US presidential election. Nature communications. 2019;10(1):1-14.

9. Allcott H, Gentzkow M. Social media and fake news in the 2016 election. Journal of economic perspectives. 2017;31(2):211-36.

10. Shin J, Jian L, Driscoll K, Bar F. The diffusion of misinformation on social media: Temporal pattern, message, and source. Computers in Human Behavior. 2018;83:278-287.

11. Cinelli M, Morales GDF, Galeazzi A, Quattrociocchi W, Starnini M. The echo chamber effect on social media. Proceedings of the National Academy of Sciences. $2021 ; 118(9)$.

12. Törnberg P. Echo chambers and viral misinformation: Modeling fake news as complex contagion. PloS one. 2018;13(9):e0203958.

13. Allcott H, Gentzkow M, Yu C. Trends in the diffusion of misinformation on social media. Research \& Politics. 2019;6(2):2053168019848554.

14. Daley DJ, Kendall DG. Epidemics and rumours. Nature. 1964;204(4963):1118-1118.

15. Jin F, Dougherty E, Saraf P, Cao Y, Ramakrishnan N. Epidemiological modeling of news and rumors on twitter. In: Proceedings of the 7 th workshop on social network mining and analysis; 2013. p. 1-9.

16. Bettencourt LM, Cintrón-Arias A, Kaiser DI, Castillo-Chávez C. The power of a good idea: Quantitative modeling of the spread of ideas from epidemiological models. Physica A: Statistical Mechanics and its Applications. 2006;364:513-536.

17. Nekovee M, Moreno Y, Bianconi G, Marsili M. Theory of rumour spreading in complex social networks. Physica A: Statistical Mechanics and its Applications. 2007;374(1):457-470.

18. Zhao L, Cui H, Qiu X, Wang X, Wang J. SIR rumor spreading model in the new media age. Physica A: Statistical Mechanics and its Applications. 2013;392(4):995-1003.

19. Shah D, Zaman T. Rumors in a network: Who's the culprit? IEEE Transactions on information theory. 2011;57(8):5163-5181.

20. Skaza J, Blais B. Modeling the infectiousness of Twitter hashtags. Physica A: Statistical Mechanics and its Applications. 2017;465:289-296.

21. Ahmed W, Vidal-Alaball J, Downing J, Seguí FL. COVID-19 and the 5G conspiracy theory: social network analysis of Twitter data. Journal of Medical Internet Research. 2020;22(5):e19458. 
22. Hamilton IA. 77 Phone Masts Attacked in UK Due to Coronavirus 5G Conspiracy Theory. In: Businessinsider. 2020 May 6 [Cited 2021 March 28];Available from: https://www.businessinsider.com/ 77-phone-masts-fire-coronavirus-5g-conspiracy-theory-2020-5?r=DE\& $\mathrm{IR}=\mathrm{T}$.

23. Waterson J, Hern A. At least 20 UK phone masts vandalised over false $5 \mathrm{G}$ coronavirus claims; 2020.

24. Bruns A, Harrington S, Hurcombe E. 'Corona? 5G? or both?': the dynamics of COVID-19/5G conspiracy theories on Facebook. Media International Australia. 2020;

25. Facebook. How Facebook's Fact-Checking Program Works. In: facebook journalism project. 2020 Aug 11 [Cited 2021 March 02];Available from: https://www.facebook.com/journalismproject/programs/ third-party-fact-checking/how-it-works

26. Pennycook G, Rand D. Assessing the effect of "disputed" warnings and source salience on perceptions of fake news accuracy. Social Science Research Network https://papers ssrn com/sol3/papers cfm. 2017;

27. Clayton K, Blair S, Busam JA, Forstner S, Glance J, Green G, et al. Real solutions for fake news? Measuring the effectiveness of general warnings and fact-check tags in reducing belief in false stories on social media. Political Behavior. 2020;42(4):1073-1095.

28. Mena P. Cleaning up social media: The effect of warning labels on likelihood of sharing false news on Facebook. Policy \& internet. 2020;12(2):165-183.

29. Inc T. Coronavirus: Staying safe and informed on Twitter. In: Twitter Blog. 2020 Jan 12 [Cited 2021 March 28]; Available from: https://blog.twitter.com/en_us/topics/company/2020/covid-19.html

30. Coleman K. Introducing Birdwatch, a community-based approach to misinformation; 2021 Jan 21 [Cited 2021 March 27]. Available from: https://blog.twitter.com/en_us/topics/product/2021/ introducing-birdwatch-a-community-based-approach-to-misinformation html.

31. Margolin DB, Hannak A, Weber I. Political fact-checking on Twitter: When do corrections have an effect? Political Communication. 2018;35(2):196-219.

32. Shu K, Sliva A, Wang S, Tang J, Liu H. Fake news detection on social media: A data mining perspective. ACM SIGKDD explorations newsletter. 2017;19(1):22-36.

33. Tacchini E, Ballarin G, Della Vedova ML, Moret S, de Alfaro L. Some like it hoax: Automated fake news detection in social networks. arXiv preprint arXiv:170407506. 2017; .

34. Tschiatschek S, Singla A, Gomez Rodriguez M, Merchant A, Krause A. Fake news detection in social networks via crowd signals. In: Companion Proceedings of the The Web Conference 2018; 2018. p. 517-524.

35. Kumar KK, Geethakumari G. Detecting misinformation in online social networks using cognitive psychology. Human-centric Computing and Information Sciences. 2014;4(1):1-22. 
36. Beckley R, Weatherspoon C, Alexander M, Chandler M, Johnson A, Bhatt GS. Modeling epidemics with differential equation. Tennessee State University Internal Report. 2013;

37. Zhao L, Wang J, Chen Y, Wang Q, Cheng J, Cui H. SIHR rumor spreading model in social networks. Physica A: Statistical Mechanics and its Applications. 2012;391(7):2444-2453.

38. Nicho J. The SIR Epidemiology Model in Predicting Herd Immunity. Undergraduate Journal of Mathematical Modeling: One+ Two. 2010;2(2):8.

39. Peri SSS, Chen B, Dougall AL, Siemens G. Towards understanding the lifespan and spread of ideas: epidemiological modeling of participation on Twitter. In: Proceedings of the Tenth International Conference on Learning Analytics \& Knowledge; 2020. p. 197-202.

40. Tambuscio M, Ruffo G, Flammini A, Menczer F. Fact-checking effect on viral hoaxes: A model of misinformation spread in social networks. In: Proceedings of the 24th international conference on World Wide Web; 2015. p. 977-982.

41. Cheng JJ, Liu Y, Shen B, Yuan WG. An epidemic model of rumor diffusion in online social networks. The European Physical Journal B. 2013;86(1):1-7.

42. R Core Team. R: A Language and Environment for Statistical Computing; 2020. Available from: https://www.R-project.org/.

43. Jenness SM, Goodreau SM, Morris M. EpiModel: an R package for mathematical modeling of infectious disease over networks. Journal of statistical software. $2018 ; 84$.

44. Garrett RK, Nisbet EC, Lynch EK. Undermining the corrective effects of media-based political fact checking? The role of contextual cues and naïve theory. Journal of Communication. 2013;63(4):617-637.

45. Walter N, Cohen J, Holbert RL, Morag Y. Fact-checking: A meta-analysis of what works and for whom. Political Communication. 2020;37(3):350-375.

46. Brennen JS, Simon F, Howard PN, Nielsen RK. Types, sources, and claims of COVID-19 misinformation. Reuters Institute. 2020;7:3-1.

47. Banda JM, Tekumalla R, Wang G, Yu J, Liu T, Ding Y, et al. A large-scale COVID-19 Twitter chatter dataset for open scientific research-an international collaboration. arXiv preprint arXiv:200403688. 2020;.

48. Stempel C, Hargrove T, Stempel III GH. Media use, social structure, and belief in 9/11 conspiracy theories. Journalism \& Mass Communication Quarterly. 2007;84(2):353-372.

49. Mann RP, Faria J, Sumpter DJ, Krause J. The dynamics of audience applause. Journal of The Royal Society Interface. 2013;10(85):20130466.

50. Munz P, Hudea I, Imad J, Smith RJ. When zombies attack!: mathematical modelling of an outbreak of zombie infection. Infectious disease modelling research progress. 2009;4:133-150.

51. Rodrigues HS. Application of SIR epidemiological model: new trends. arXiv preprint arXiv:161102565. 2016; 
52. Giachanou A, Crestani F. Like it or not: A survey of twitter sentiment analysis methods. ACM Computing Surveys (CSUR). 2016;49(2):1-41.

53. Krishnan S, Chen M. Identifying tweets with fake news. In: 2018 IEEE International Conference on Information Reuse and Integration (IRI). IEEE; 2018. p. 460-464.

54. Buntain C, Golbeck J. Automatically identifying fake news in popular twitter threads. In: 2017 IEEE International Conference on Smart Cloud (SmartCloud). IEEE; 2017. p. 208-215.

55. Shao C, Ciampaglia GL, Varol O, Flammini A, Menczer F. The spread of fake news by social bots. arXiv preprint arXiv:170707592. 2017;96:104.

56. Selivanov D, Wang Q. text2vec: Modern text mining framework for $\mathrm{r}$. Computer software manual](R package version 040 ) Retrieved from https://CRAN $\mathrm{R}$-project org/package $=$ text2vec. 2016;

57. Davis CA, Varol O, Ferrara E, Flammini A, Menczer F. Botornot: A system to evaluate social bots. In: Proceedings of the 25th international conference companion on world wide web; 2016. p. 273-274.

58. King AA, Domenech de Cellès M, Magpantay FM, Rohani P. Avoidable errors in the modelling of outbreaks of emerging pathogens, with special reference to Ebola. Proceedings of the Royal Society B: Biological Sciences. 2015;282(1806):20150347.

59. Endsley MR. Combating information attacks in the age of the Internet: new challenges for cognitive engineering. Human factors. 2018;60(8):1081-1094.

60. Ognyanova K, Lazer D, Robertson RE, Wilson C. Misinformation in action: Fake news exposure is linked to lower trust in media, higher trust in government when your side is in power. Harvard Kennedy School Misinformation Review. 2020;

61. Levy N. The bad news about fake news. Social epistemology review and reply collective. 2017;6(8):20-36.

62. Goldberg ZJ, Richey S. Anti-vaccination beliefs and unrelated conspiracy theories. World Affairs. 2020;183(2):105-124.

63. Shu K, Mahudeswaran D, Wang S, Lee D, Liu H. Fakenewsnet: A data repository with news content, social context, and spatiotemporal information for studying fake news on social media. Big Data. 2020;8(3):171-188.

64. Lewandowsky S, Ecker UK, Seifert CM, Schwarz N, Cook J. Misinformation and its correction: Continued influence and successful debiasing. Psychological science in the public interest. 2012;13(3):106-131.

65. Roediger III HL, Marsh EJ. The positive and negative consequences of multiple-choice testing. Journal of Experimental Psychology: Learning, Memory, and Cognition. 2005;31(5):1155.

66. Vosoughi S, Roy D, Aral S. The spread of true and false news online. Science. 2018;359(6380):1146-1151.

67. Pennycook G, Rand DG. The psychology of fake news. Trends in cognitive sciences. 2021; in press;doi:10.1016/j.tics.2021.02.007.

68. Jolley D, Douglas KM. The social consequences of conspiracism: Exposure to conspiracy theories decreases intentions to engage in politics and to reduce one's carbon footprint. British Journal of Psychology. 2014;105(1):35-56. 
A

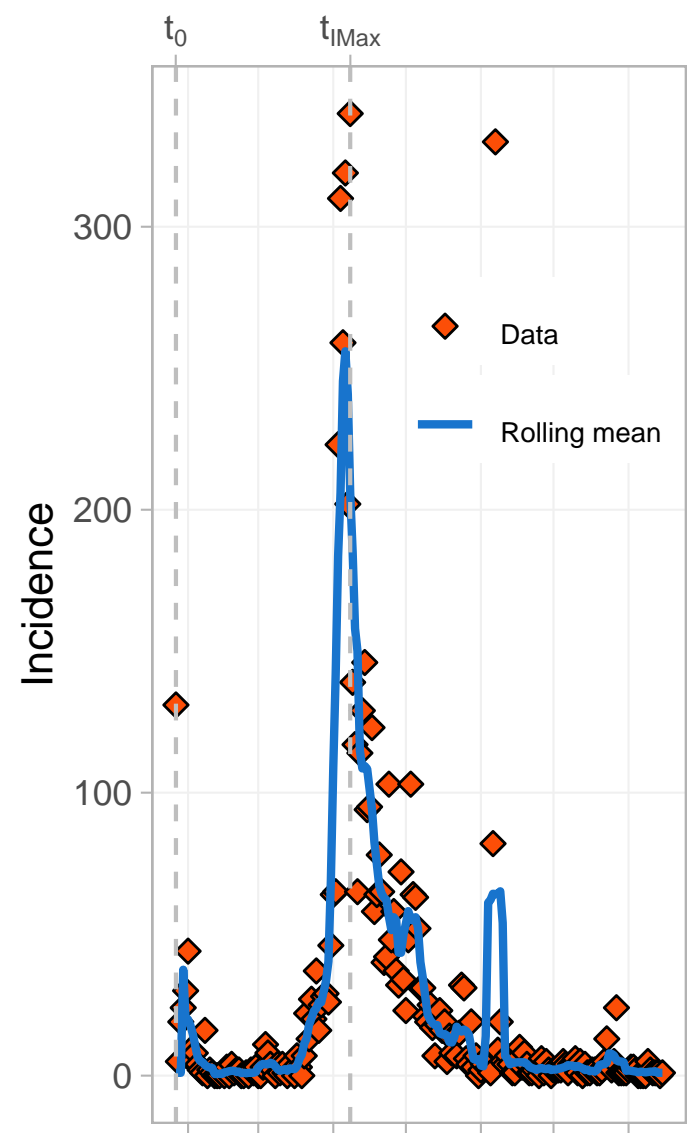

Feb Mar Apr May Jun Jul Aug Date
B

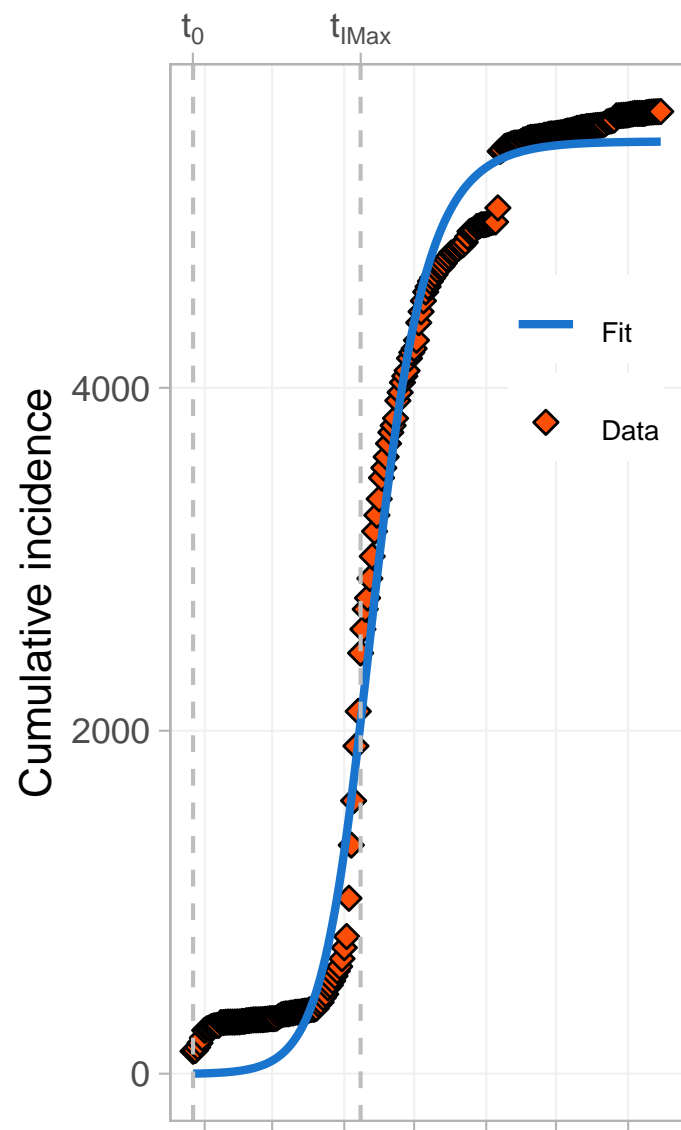

Feb Mar Apr May Jun Jul Aug Date

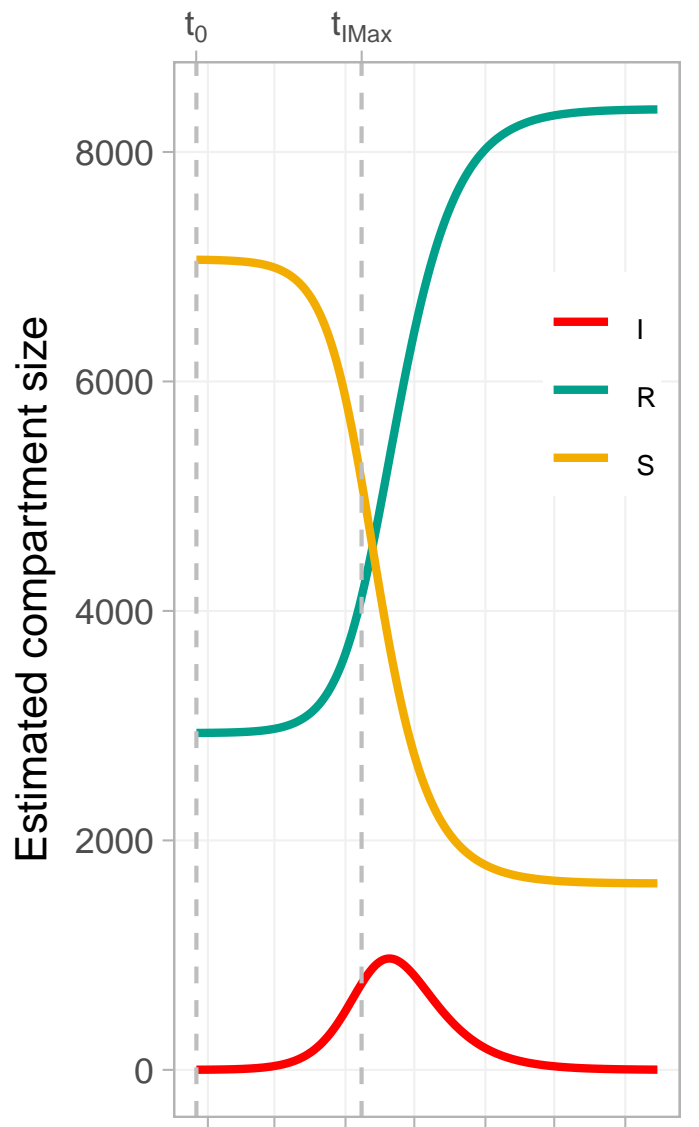

Feb Mar Apr May Jun Jul Aug Date 


\begin{tabular}{|c|c|c|c|c|c|c|}
\hline Model & $\bar{\delta}$ & $\gamma$ & $\zeta$ & $\begin{array}{l}\text { Total Num- } \\
\text { ber of Infec- } \\
\text { tions }\end{array}$ & $\begin{array}{l}\text { Total Num- } \\
\text { ber of } \\
\text { Prevented } \\
\text { Infections }\end{array}$ & $I P_{f}$ \\
\hline basic & Inf & 0 & 0 & 5435 & 0 & 0.770 \\
\hline \multirow{9}{*}{ fact-checking } & 14 & 0.01 & 0 & 4486 & 949 & 0.635 \\
\hline & 14 & 0.03 & 0 & 2563 & 2871 & 0.363 \\
\hline & 14 & 0.05 & 0 & 1128 & 4307 & 0.160 \\
\hline & 42 & 0.01 & 0 & 4900 & 535 & 0.694 \\
\hline & 42 & 0.03 & 0 & 3898 & 1537 & 0.552 \\
\hline & 42 & 0.05 & 0 & 3039 & 2396 & 0.430 \\
\hline & 59 & 0.01 & 0 & 5124 & 311 & 0.726 \\
\hline & 59 & 0.03 & 0 & 4566 & 869 & 0.647 \\
\hline & 59 & 0.05 & 0 & 4090 & 1345 & 0.579 \\
\hline \multirow{9}{*}{ deletion } & 14 & 0 & 0.06 & 5091 & 343 & 0.721 \\
\hline & 14 & 0 & 0.12 & 4720 & 714 & 0.669 \\
\hline & 14 & 0 & 0.25 & 3819 & 1616 & 0.541 \\
\hline & 42 & 0 & 0.06 & 5097 & 337 & 0.722 \\
\hline & 42 & 0 & 0.12 & 4736 & 699 & 0.671 \\
\hline & 42 & 0 & 0.25 & 3880 & 1555 & 0.549 \\
\hline & 59 & 0 & 0.06 & 5121 & 314 & 0.725 \\
\hline & 59 & 0 & 0.12 & 4793 & 642 & 0.679 \\
\hline & 59 & 0 & 0.25 & 4057 & 1378 & 0.575 \\
\hline \multirow{27}{*}{ mixed } & 14 & 0.01 & 0.06 & 4041 & 1393 & 0.572 \\
\hline & 14 & 0.01 & 0.12 & 3555 & 1880 & 0.503 \\
\hline & 14 & 0.01 & 0.25 & 2355 & 3080 & 0.333 \\
\hline & 14 & 0.03 & 0.06 & 1990 & 3445 & 0.282 \\
\hline & 14 & 0.03 & 0.12 & 1438 & 3996 & 0.204 \\
\hline & 14 & 0.03 & 0.25 & 560 & 4875 & 0.079 \\
\hline & 14 & 0.05 & 0.06 & 757 & 4678 & 0.107 \\
\hline & 14 & 0.05 & 0.12 & 494 & 4941 & 0.070 \\
\hline & 14 & 0.05 & 0.25 & 203 & 5232 & 0.029 \\
\hline & 42 & 0.01 & 0.06 & 4524 & 911 & 0.641 \\
\hline & 42 & 0.01 & 0.12 & 4123 & 1312 & 0.584 \\
\hline & 42 & 0.01 & 0.25 & 3193 & 2242 & 0.452 \\
\hline & 42 & 0.03 & 0.06 & 3477 & 1957 & 0.492 \\
\hline & 42 & 0.03 & 0.12 & 3048 & 2387 & 0.432 \\
\hline & 42 & 0.03 & 0.25 & 2150 & 3285 & 0.304 \\
\hline & 42 & 0.05 & 0.06 & 2632 & 2803 & 0.373 \\
\hline & 42 & 0.05 & 0.12 & 2242 & 3192 & 0.318 \\
\hline & 42 & 0.05 & 0.25 & 1524 & 3911 & 0.216 \\
\hline & 59 & 0.01 & 0.06 & 4802 & 633 & 0.680 \\
\hline & 59 & 0.01 & 0.12 & 4469 & 966 & 0.633 \\
\hline & 59 & 0.01 & 0.25 & 3742 & 1693 & 0.530 \\
\hline & 59 & 0.03 & 0.06 & 4242 & 1193 & 0.601 \\
\hline & 59 & 0.03 & 0.12 & 3918 & 1517 & 0.555 \\
\hline & 59 & 0.03 & 0.25 & 3247 & 2188 & 0.460 \\
\hline & 59 & 0.05 & 0.06 & 3780 & 1655 & 0.535 \\
\hline & 59 & 0.05 & 0.12 & 3479 & 1956 & 0.493 \\
\hline & 59 & 0.05 & 0.25 & 2883 & 2552 & 0.408 \\
\hline
\end{tabular}

